Correction

\title{
Correction: Systematic discovery of drug action mechanisms by an integrated chemical genomics approach: identification of functional disparities between azacytidine and decitabine
}

\author{
Yao-Yu Hsieh ${ }^{1,2,5}$, Tsui-Chin Huang ${ }^{1,3}$, Hsiang-Ling Lo ${ }^{1,3}$, Jyun-Yan Jhan ${ }^{1,3}$, Shui- \\ Tein Chen ${ }^{1,4}$ and Pei-Ming Yang ${ }^{1,3}$ \\ ${ }^{1}$ PhD Program for Cancer Biology and Drug Discovery, College of Medical Science and Technology, Taipei Medical University \\ and Academia Sinica, Taipei, Taiwan \\ ${ }^{2}$ Division of Hematology and Oncology, Shuang Ho Hospital, Taipei Medical University, Taipei, Taiwan \\ ${ }^{3}$ Graduate Institute of Cancer Biology and Drug Discovery, College of Medical Science and Technology, Taipei Medical \\ University, Taipei, Taiwan \\ ${ }^{4}$ Institute of Biological Chemistry, Academia Sinica, Taipei, Taiwan \\ ${ }^{5}$ Department of Internal Medicine, Division of Hematology and Oncology, School of Medicine, College of Medicine, Taipei \\ Medical University, Taipei, Taiwan \\ Published: August 17, 2018
}

Copyright: Hsieh et al. This is an open-access article distributed under the terms of the Creative Commons Attribution License 3.0 (CC BY 3.0), which permits unrestricted use, distribution, and reproduction in any medium, provided the original author and source are credited.

This article has been corrected: The author affiliation is given below:

\section{Yao-Yu Hsieh ${ }^{1,2,5}$}

${ }^{5}$ Department of Internal Medicine, Division of Hematology and Oncology, School of Medicine, College of Medicine, Taipei Medical University, Taipei, Taiwan

Original article: Oncotarget. 2016; 7:27363-27378. https://doi.org/10.18632/oncotarget.8455 